\title{
Alarming triad of progressive hoarseness in a male smoker
}

\author{
Nur Ain Nabila Za'im, Mawaddah Azman \\ Nabila Za'im NA, Azman M. Alarming triad of progressive hoarseness in a male smoker. Malays Fam Physician. 2021;16(3);119-122. \\ https://doi.org/10.51866/cr1163
}

\section{Keywords:}

Hoarseness, laryngeal

cancer, glottic carcinoma,

smoker, red flags.

\section{Authors:}

\section{Mawaddah Azman \\ (Corresponding author) MS}

Department of Otorhinolaryngology and Head and Neck Surgery, Level 9 Clinical Block, Universiti Kebangsaan Malaysia Medical Centre, Jalan Yaacob Latiff, Bandar Tun Razak Cheras, Kuala Lumpur, Malaysia Email: mawaddah1504@yahoo.co

\section{Nur Ain Nabila Za'im MD}

Department of Otorhinolaryngology and Head and Neck Surgery, Level 9 Clinical Block, Universiti Kebangsaan Malaysia Medical Centre, Jalan Yaacob Latiff, Bandar Tun Razak Cheras, Kuala Lumpur, Malaysia

\begin{abstract}
Hoarseness accounts for $1 \%$ of all consultations in primary care. Suspicion of malignancy should be considered in individuals with risk factors presenting with unexplained hoarseness lasting more than two weeks. A significant number of patients with laryngeal cancer present at an advanced stage due to lack of awareness regarding vocal health. It is important to educate both the public and primary care health providers concerning laryngeal cancer. We present the case of an 81-yearold male smoker who presented to us with a six-month history of progressive hoarseness. He was initially treated in two primary and one secondary care centres, where a diagnosis of laryngeal cancer was not considered. Careful assessment in our centre managed to determine a diagnosis of T3N0M0 glottic carcinoma. We will discuss this alarming triad of progressive hoarseness in a male smoker to help primary care physicians streamline their thoughts and identify red flags in a hoarse patient.
\end{abstract}

\section{Introduction}

Hoarseness, or alteration of voice, accounts for $1 \%$ of all consultations in primary care. Its common causes are acute and chronic laryngitis, accounting for $42.1 \%$ and 9.7\% respectively, followed by functional dysphonia, benign and malignant tumours, neurogenic factors and aging. ${ }^{1}$ Any patient complaining of persistent or progressive hoarseness lasting more than two weeks with risk factors should prompt immediate investigation and referral to a tertiary centre to exclude laryngeal cancer., ${ }^{2,3}$ Despite its early presentation with a striking symptom such as hoarseness, delayed referral to an otolaryngologist is commonly observed in laryngeal cancer, such as seen in our case.

\section{Case Presentation}

An 81-year-old Chinese male who was an active smoker was referred from a secondary centre with complaint of progressive hoarseness over the past six months, with no other associated symptoms. He visited two primary care clinics prior to his presentation, where two courses of antibiotics were prescribed over a period of two months. Subsequently, he was referred to a secondary centre where an otorhinolaryngology consult was obtained. Neck examination revealed a right anterior neck swelling, measuring $2 \times 2 \mathrm{~cm}$, which moved with deglutition.
Initial examination by the otolaryngologist at the secondary centre revealed left unilateral vocal fold paralysis with no suspicion of malignancy. A contrasted CT scan showed an enlarged left thyroid gland causing tracheal deviation (Figure 1). The patient was subsequently referred to an otolaryngologist at a tertiary centre for further management of a benign thyroid mass.

Endoscopic examination of the larynx at the tertiary centre showed left vocal fold immobility with an irregular mass on the left vocal fold (Figure 2). Histopathological examination of the left vocal cord and subglottic mass showed poorly differentiated squamous cell carcinoma. Fine needle aspiration cytological examination of the left thyroid lobe revealed benign follicular nodules. After further counselling, the patient underwent total laryngectomy, left hemithyroidectomy and left selective neck dissection (levels I, II and III) for T3N0M0 glottic cancer with left follicular thyroid nodule. Histopathological examination of the surgical specimen revealed squamous cell carcinoma with clear margins. 


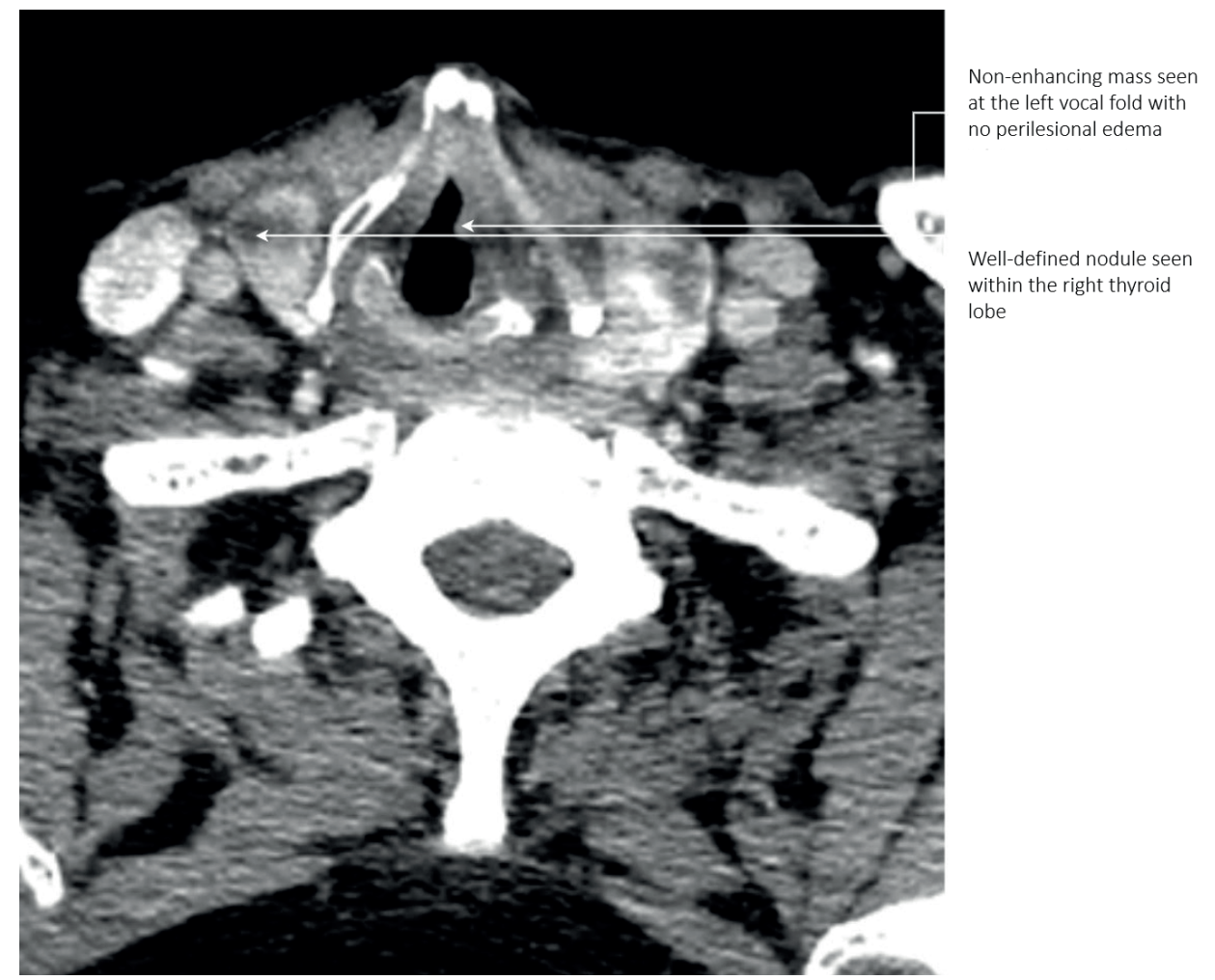

Figure 1. Axial cut contrast CT neck of an 81-year-old man with hoarseness showing a nonenhancing mass arising from the left vocal fold, which was missed by the secondary centre. A corresponding well-defined, non-enhancing thyroid nodule was seen arising from the right hemithyroid. In this instance, the initial finding of left vocal fold immobility did not correlate with a thyroid pathology.

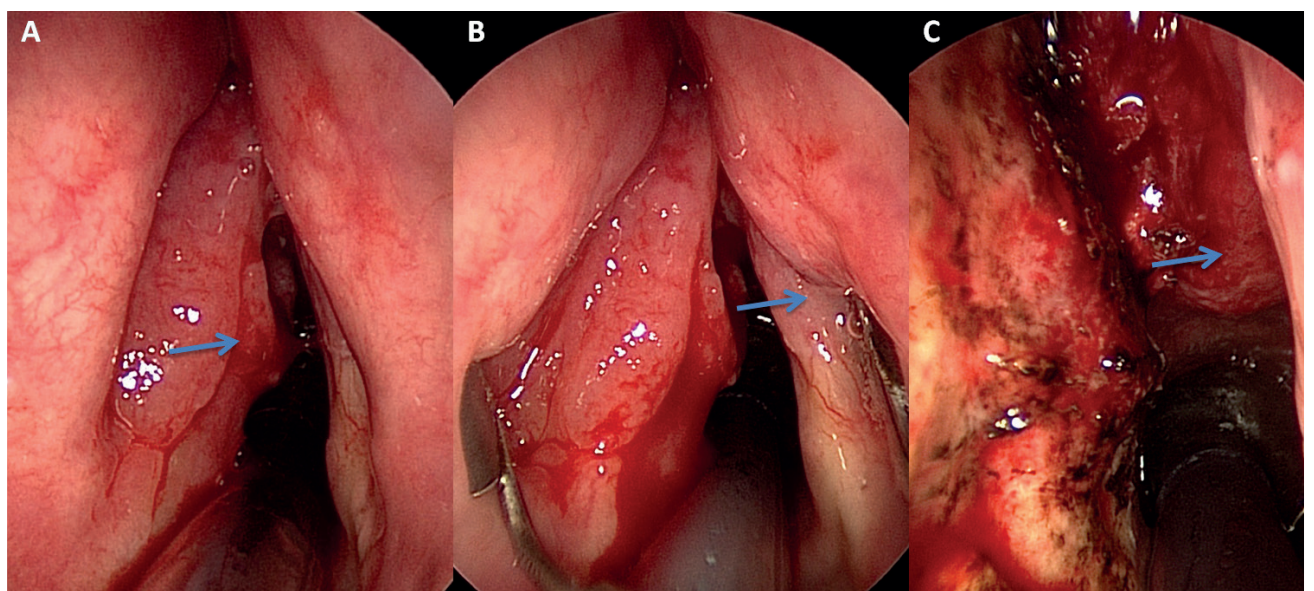

Figure 2. Endoscopic images of the vocal cords of an 81-year-old man with hoarseness showing a fungating and friable mass arising from the left true cord (A: blue arrow) extending to the anterior commissure and right true cord (B: blue arrow). Following laser excision of the vocal cord lesions, subglottic extension (C: blue arrow) was visible, indicating an advanced staged tumour.

\section{Discussion}

Laryngeal cancer is the second most common head and neck cancer in Malaysia, more commonly seen in patients of advanced age, with a mean of 59 years. ${ }^{2}$ Principally laryngeal cancer is a disease of men, and is highly associated with smoking and alcohol consumption. ${ }^{2,3}$ In Malaysia, the majority of laryngeal cancer patients are Chinese, who account for $54 \%$ of cases, followed by Indians (23\%) and Malays (19\%). ${ }^{3}$ The reported gender distribution is 7.6:1 male to female, with an obvious predilection to the male gender. ${ }^{3}$ Population-based studies of laryngeal cancer in other parts of the world have reported similar predilections to men, with male to 
female ratios between 8.4:1.6 and 30:1.3.4 The higher risk of developing laryngeal cancer is directly related to the increased prevalence of unhealthy habits like tobacco smoking and alcohol consumption among males. A recent study documented an increased risk in women who were first-hand and passive smokers, considerably reducing the male to female ratio. ${ }^{5}$

The main causative factors for laryngeal cancer are tobacco smoking and alcohol consumption. Various studies have shown that these factors have a synergistic effect on the development of laryngeal cancer. ${ }^{2-5}$ It is well documented that derivatives from commercially prepared tobacco act as carcinogens in the epithelium of the larynx and disrupt division and proliferation of cells, triggering carcinogenesis. ${ }^{6}$ Examples of these carcinogenic derivatives include polycyclic aromatic hydrocarbons, $\mathrm{N}$-nitrosamines, aromatic amines, aldehydes and volatile organic hydrocarbons. Studies have shown that the tobacco-specific nitrosamine 4-(methylnitrosamino)-1-(3-pyridyl)-1butanone upregulates oncogenic pathways linked to head and neck cancer. ${ }^{6}$ The past decade has seen the emergence of electronic cigarettes (e-cigarettes), which $13-50 \%$ of tobacco smokers have been found to also use. $^{7}$ Although e-cigarettes are safer than tobacco products because they do not contain nitrosamines, scientific evidence regarding their carcinogenic effect is limited due to a lack of long-term data and heterogeneity in the chemical compounds found in e-cigarettes. ${ }^{7}$ Other risk factors for laryngeal cancer include gastroesophageal reflux disease $^{8}$ and certain professions. Exposure to carcinogenic agents like asbestos, textile dust and strong acid mist is associated with laryngeal cancer among miners, textile workers and workers in metal industries.

Glottic cancer, which presents in its early stage with hoarseness, accounts for nearly $90 \%$ of laryngeal cancer. ${ }^{2.3}$ A study in the United States reported that $2.2 \%$ of individuals with persistent hoarseness lasting more than four weeks were found to have laryngeal cancer. ${ }^{1}$ Another local study reported that hoarseness was the main symptom of laryngeal cancer, followed by stridor and dysphagia. ${ }^{3}$ Hoarseness has a broad range of aetiologies, varying from simple flu to malignancy, making the diagnosis of hoarseness challenging for a general practitioner. ${ }^{1}$ A history of intermittent hoarseness with a normal voice in between episodes of hoarseness, an association with upper respiratory tract infection or an exacerbation with allergy and reflux symptoms suggest benign causes of hoarseness. Conversely, persistent and progressive hoarseness is more likely to have a malignant cause. ${ }^{10}$ Presence of other symptoms and signs such as dysphagia, dyspnoea, aspiration, haemoptysis, otalgia, stridor, neck mass and weight loss are irrelevant, as they suggest advanced disease. Throat examination in a patient with laryngeal carcinoma is usually normal, making endoscopic visualisation of the larynx by an otolaryngologist, the only way to detect early lesions.

Two local studies conducted in Malaysia reported that a significant percentage of patients with laryngeal cancer (75\%) presented in an advanced stage requiring aggressive surgical treatment with significant postoperative functional morbidities.,3 These local scenarios raise concerns over late presenting patients with laryngeal cancer seeking medical advice. We would like to educate the public and primary health care providers regarding the importance of early detection and prompt referral to a tertiary centre for further evaluation. A patient presenting with persistent or progressive hoarseness, with risk factors such as being male and a chronic smoker or alcohol consumer, should raise suspicions of an underlying malignant process. The presence of this alarming triad warrants prompt and careful evaluation by a trained otolaryngologist. Early referral increases the likelihood of conservative treatment protocols with superior functional and oncologic outcomes. 


\section{How does this paper make a difference to general practice?}

- Red flag triad in a patient presenting with hoarseness includes:

- Persistent or progressive hoarseness lasting more than two weeks

- Male gender

- Presence of a risk factor such as tobacco or alcohol consumption

- Presence of these red flags mandates referral to an otolaryngologist for prompt endoscopic examination (Figure 3).

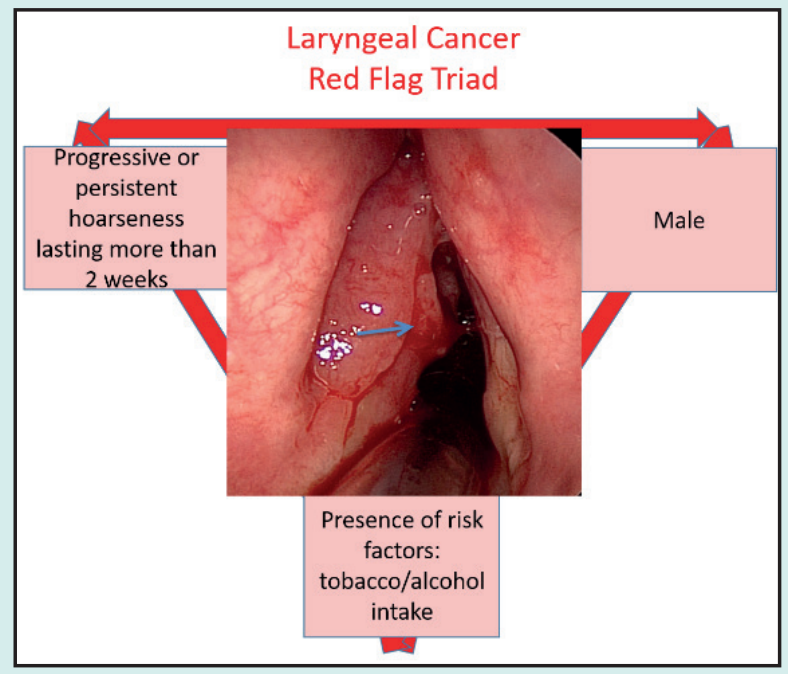

- In laryngeal cancer, early referral to an otolaryngologist increases the likelihood of conservative treatment protocols with superior functional and oncologic outcomes.

\section{References}

1. Reiter R, Hoffmann TK, Pickhard A, et al. Hoarseness-Causes and Treatments. Dtsch Arztebl Int. 2015;112(19):329-337.

2. Abdullah K, Raja Lope Ahmad RA, Asha'ari ZA, et al. An Outcome of Surgically Treated Head and Neck Cancer in One of the Tertiary Referral Center in the East Coast of Malaysia: A 6-year Retrospective Analysis. Malays J Med Sci. 2014 Jul;21(4):28-36.

3. Sani A, Said H, Lokman S. Carcinoma of the Larynx in Malaysia. Med J Malaysia. 1992 Dec;47(4):297-302.

4. Markou K, Christoforidou A, Karasmanis I, et al. Laryngeal Cancer: Epidemiological Data from Northern Greece and Review of the Literature. Hippokratia. 2013 Oct-Dec;17(4), 313-318.
5. Majszyk D, Bruzgielewicz A, OsuchWójcikiewicz E, et al. Gender-related Incidence, Risk Factors Exposure and Survival Rates of Laryngeal Cancers - The 10-years Analysis of Trends from One Institution. Otolaryngol Pol. 2019;73(3):6-10.

6. Doukas SG, Vageli DP, Lazopoulos G, et al. The Effect of NNK, A Tobacco Smoke Carcinogen, on the miRNA and Mismatch DNA Repair Expression Profiles in Lung and Head and Neck Squamous Cancer Cells. Cells. 2020;9(4):1031.

7. Szukalska M, Szyfter K, Florek E, et al.

Electronic Cigarettes and Head and Neck Cancer Risk-Current State of Art. Cancers (Basel). 2020;12(11):3274.
8. Anis MM, Razavi MM, Xiao X, et al. Association of Gastroesophageal Reflux Disease and Laryngeal Cancer. World J Otorhinolaryngol Head Neck Surg. 2018;4(4):278-281.

9. Bayer O, Cámara R, Zeissig SR, et al. Occupation and Cancer of the Larynx: A Systematic Review and Meta-analysis. Eur Arch Otorhinolaryngol. 2016;273(1):9-20.

10. Mau T. Diagnostic Evaluation and Management of Hoarseness. Med Clin North Am. 2010;94(5):945-960. 